\title{
Spatial gamut mapping among non-inclusive gamuts
}

\author{
Javier Vazquez-Corral and Marcelo Bertalmío \\ Universitat Pompeu Fabra, E-08018, Barcelona, Spain (e-mails: \{javier.vazquez, \\ marcelo.bertalmio\}@upf.edu)
}

\begin{abstract}
Gamut mapping transforms the color gamut of an image to that of a target device. Two cases are usually considered: gamut reduction (target gamut smaller than source gamut), and gamut extension (target gamut larger than the source gamut). Less attention is devoted to the more general case, when neither gamut is fully included in the other. In this work we unify and expand two recent methods for gamut extension and reduction, so as to simultaneously perform both forms of gamut mapping in different regions of the same image without introducing color artifacts or halos. We demonstrate the usefulness of this approach for the traditional gamut mapping problem, and also how the proposed method can be used to adapt the color palette of an image so that it is closer to that of a given reference image. Results are compared with the state-of-the-art and validated through user tests and objective metrics.
\end{abstract}

Keywords: Gamut mapping, gamut extension, gamut reduction, color coherence.

\section{Introduction}

The term color gamut refers either to the set of colors that are present in an image or the set of colors that a device can reproduce. Gamut mapping is the process by which an image is modified so that its colors are adapted to the 5 gamut of a given output device, and it can take the form of gamut reduction, when the target gamut is included in the source gamut, or gamut extension, when the target gamut is larger than and includes the source gamut.

Preprint submitted to Journal of ${ }^{A} T_{E} X$ Templates

May 14, 2018 
Despite the fact that the literature on the subject of gamut mapping is quite extensive (see [1]), very little attention has been paid to the more general problem, when the intersection of source and target gamuts does not coincide with either of them (i.e. neither gamut is fully included in the other). This is not an unusual problem [2, 3, 4] and a general form of gamut mapping would therefore be useful in these situations.

In this work we introduce a method to solve this problem by performing gamut reduction on some parts of the image and gamut extension on the rest, and to the best of our knowledge this is the first work to tackle simultaneously both forms of gamut mapping. Our approach can be used not only for gamut mapping but also in order to make an image more similar (or coherent) in terms of color with respect to a given reference. In order to validate the proposed 20 method we perform a psychophysical study and we also compute quantitative measures for the results of the different applications.

The rest of the paper is organized as follows. In the next section we present some related works to our approach. In section III we present our method. Later on, in section IV we show the results of our approach on the typical gamut mapping problem. In section $\mathrm{V}$ we focus on the color coherence application. Finally, in section VI we sum up the conclusions.

\section{Related work}

\subsection{Gamut Mapping}

Plenty of Gamut Mapping Algorithms (GMAs) exist in the literature and the interested reader is referred to the excellent book by Morovic [1]. GMAs are usually divided into Gamut Reduction (GR) and Gamut Extension (GE) algorithms. We can further divide them into global and spatial algorithms. Global algorithms [5, 6, 7, perform point-to-point matching, and therefore a particular color value will be reproduced with the same value regardless of its location in the image. On the other hand, spatial algorithms [8, 9, 10] take into account the spatial color information of the original image. 
Let us present in more detail some of these algorithms. Regarding gamut reduction, current methods perform either gamut clipping or gamut compression. In gamut clipping, all the colors that are out of the target gamut are projected to 40 the border of the gamut following some criteria, while all the in-gamut colors are left untouched. For example, the HPMINDE method [5] proposes to minimize the $\Delta E$ measure following lines of identical hue, while Zamir et al. [10] propose to perform a perceptually-based image energy minimization to move all the outof-gamut pixels to the gamut boundary. On the other hand, gamut compression algorithms [11, 12 modify all the colors of the image (both those inside and outside of the target gamut) in order to preserve the gradient information of the out-of-gamut pixels. However, gamut compression methods usually show a clear lack of saturation. Some approaches [13, 14] define a knee region on the border of the gamut to obtain the advantages of the gamut compression procedure while not performing an excessive desaturation. Based on the same idea, there exist local methods [8, 9, 15] that follow a three-stage pipeline: first, they apply a global gamut clipping method, second, they add back the high frequency image detail (obtained by a spatial filter), and finally, they perform a further gamut clipping to prevent the spatial filtering operation to place a few pixels outside the destination gamut.

The literature regarding gamut extension is much more limited than that of gamut reduction. In fact, the most simple solution to perform gamut extension is to take any compression gamut reduction algorithm and use it in the reverse direction [16. However, this approach may output images with unatural colors for well-known objects such as grass, sky, etcetera. To avoid this problem some methods 17, 18, perform user studies to learn the gamut extension trasnsforms. Other methods label the data in different categories in order to perform gamut extension differently in each category. For example, [19, 20] are defined to preserve skin tones, while 21] performs a different extension depending on the chroma of the object, and 22] categorizes different memory colors. In [6] different methods are proposed and evaluated. There are very few local gamut extension algorithms. The method presented in [15] performs a two-stage process, first 
extending the source gamut using a non-linear hue-varying transform, and second, applying an image-dependent chroma smoothing operation. Finally, the methods of [10, 23 run a perceptually-based image energy minimization.

The approach presented in this paper is different in nature to all the aforementioned methods, since our goal is to simultaneously perform in the same image both gamut reduction and gamut extension. To the best of our knowledge this is the first approach of this kind.

\subsection{Color coherence}

Many works have been devoted to the general problem of color transfer since it was introduced in the literature [24]. In general, color transfer is defined as the imposition of the color characteristics of one image on another image. Some of the most influential works are [24, 25, 26]. Similarly, there are works that deal with the color stabilization problem, where two images that share the same content are color-matched [27, 28, 29, 30. Hacohen et al. 31 extend the pair-wise color stabilization problem to image stacks, and they call it color consistency. To do so, they construct a graph that relates which image parts are matched among the images (for example, it relates the grass appearing in one image to the grass appearing in all the other images). Then, they optimize a quadratic cost function on the entire graph.

In this paper we propose to solve a related but different problem to the aforementioned ones: color coherence. Color coherence aims at modifying a source image in order to make its colors consistent with the colors of an unrelated

90 second image but without modifying the general look of the first image. Color coherence is more general than color stabilization/color consistency as it does not require shared content between the images, and, at the same time, it is more restricted than color transfer since the look of the original image cannot be modified. 


\section{Proposed method for spatial gamut mapping among non-inclusive gamuts}

\subsection{Basis of our approach: variational method for gamut mapping}

In 32 the authors introduced a method for color enhancement based on some visual perception principles where given an initial image, an enhanced

100

$$
E(I)=\frac{\alpha}{2} \sum_{x}\left(I(x)-\frac{1}{2}\right)^{2}-\frac{\gamma}{2} \sum_{x} \sum_{y} w(x, y)|I(x)-I(y)|+\frac{\beta}{2} \sum_{x}\left(I(x)-I_{0}(x)\right)^{2},
$$

where $I$ is a color channel $(R, G$ or $B)$ in the range $[0,1], I_{0}$ is the original image channel, $x$ and $y$ are pixel locations, $w(x, y)$ is a normalized Gaussian kernel of standard deviation $\sigma$, and $\alpha, \beta$ and $\gamma$ are constant weights.

The constant $\alpha$ is always positive, therefore minimizing $E(I)$ favors minimizing the differences with respect to a mean value of $1 / 2$. The constant $\beta$ is also positive, so the minimization of $E(I)$ favors not departing too much away from the original image. We can see the second term of the functional, $\sum_{x} \sum_{y} w(x, y)|I(x)-I(y)|$, as a measure of local contrast. Therefore, if $\gamma$ is positive, minimizing $E(I)$ will increase the contrast, while if $\gamma<0$ then the minimization of the energy functional will reduce the contrast, as pointed out in 33 .

The top row of Figure 1 illustrates this point. The image on the left is the original, the middle image has been obtained by minimizing Eq. (1) for some value $\gamma>0$ (notice the enhanced contrast), and the image on the right was obtained with the same procedure but uisng a negative value for $\gamma$ (here the contrast has been reduced).

The bottom row of Figure 1 depicts the chromaticity diagrams for the images above, and corroborates that with $\gamma>0$ the colors become more saturated while the opposite happens for $\gamma<0$. We can see then the potential of this method to be used for gamut mapping, choosing $\gamma>0$ for gamut extension and $\gamma<0$ when gamut reduction is needed. That was precisely the approach followed in [10, 

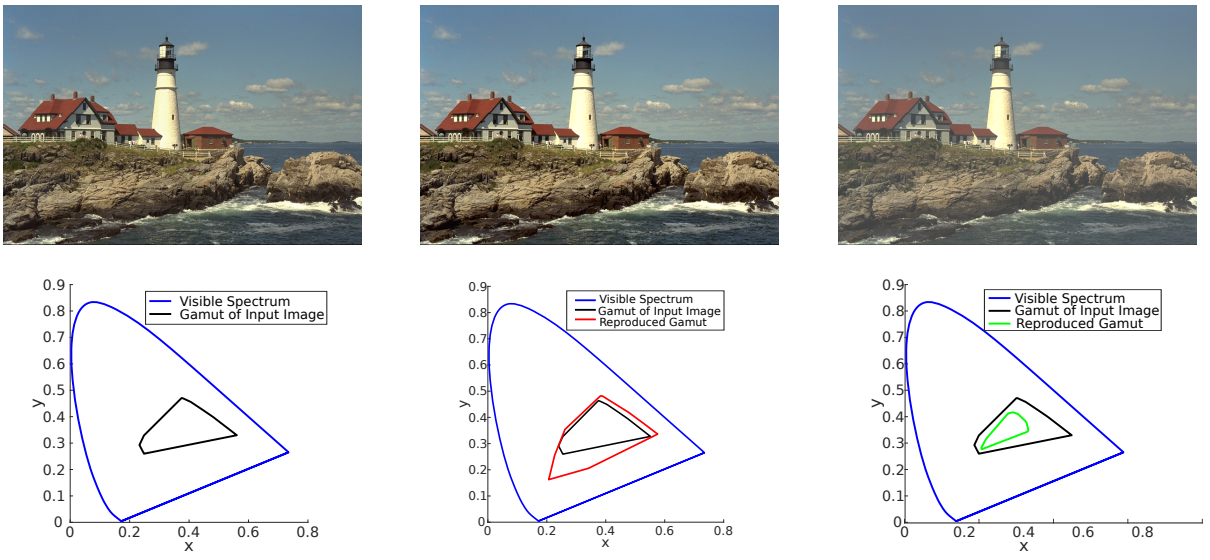

Figure 1: Example images and their corresponding gamut plots. Left column: input image. Middle column: extended-gamut image. Right column: reduced-gamut image.

where the authors replaced with the global mean average value $\mu$ the value of $1 / 2$ in the first term of the functional and then minimized the energy functional by iterating

$$
I^{k+1}(x)=\frac{I^{k}(x)+\Delta t\left(\alpha \mu+\beta I_{0}(x)+\frac{\gamma}{2} R_{I^{k}}(x)\right)}{1+\Delta t(\alpha+\beta)},
$$

125

where the initial condition is $I^{k=0}(x)=I_{0}(x), R_{I^{k}}(x)$ indicates the contrast function

$$
R_{I^{k}}(x)=\frac{\sum_{y} w(x, y) s\left(I^{k}(x)-I^{k}(y)\right)}{\sum_{y} w(x, y)}
$$

and the slope function $s(\cdot)$ is a regularized approximation of the sign function (in [32] this approximating function is chosen as a polynomial of degree seven).

Fig. $2 \mathrm{~b}$ shows that for gamut reduction $(\gamma<0)$, as the absolute value of $\gamma$ is increased the resulting gamut is reduced more. An analogous behaviour can be shown for the gamut extension case, where $\gamma>0$ and higher $\gamma$ values result in larger gamuts.

\subsection{Proposed approach}

Let us consider a source image $I_{s}$ and a target image $I_{t}$, with respective $3 \mathrm{D}$ gamuts $G_{s}$ and $G_{t}$ that are non-inclusive, as shown in Fig. 3 We can partition 


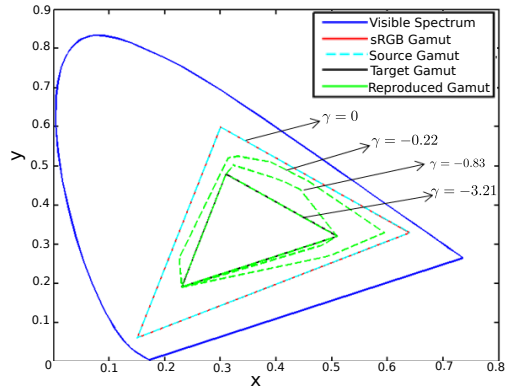

(a)

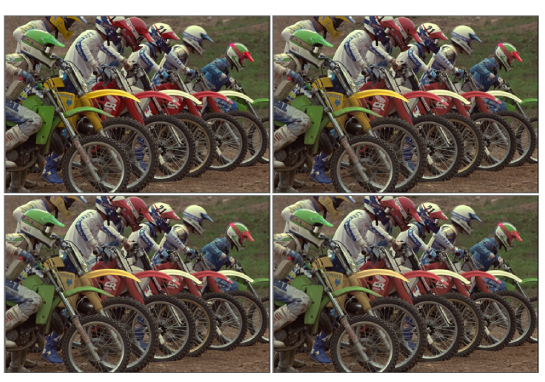

(b)

Figure 2: GR approach. (a) Gamuts on chromaticity diagram. (b) Top left: original image. Top right: $\gamma=-0.22$. Bottom left: $\gamma=-0.83$. Bottom right: $\gamma=-3.21$.

the gamuts into regions: $\Omega=G_{t} \backslash G_{s}, \Phi=G_{t} \cap G_{s}, \Psi=G_{s} \backslash G_{t}$. Furthermore, we split the gamut intersection $\Phi$ into two parts, where $\Phi_{1}$ covers the colors closer to $\Psi$ and $\Phi_{2}$ those closer to $\Omega$. Formally, the two sub-regions $\Phi_{1}$ and $\Phi_{2}$ are defined as follows:

$$
\begin{aligned}
& \Phi_{1}=\{z \in \Phi \mid d(z, \Psi) \leq d(z, \Omega)\}, \\
& \Phi_{2}=\{z \in \Phi \mid d(z, \Psi)>d(z, \Omega)\},
\end{aligned}
$$

where $d(z, \Psi)=\min _{t \in \Psi} d(z, t), d(z, \Omega)=\min _{t \in \Omega} d(z, t)$, and $d(z, t)$ is the Euclidean distance between $z$ and $t$.

In order to solve the gamut mapping problem, the goal is to modify $I_{s}$ by mapping its gamut $G_{s}$ to $G_{t}$. For this we propose the following approach: perform gamut reduction in $\Psi$ and (to a lesser extent) in $\Phi_{1}$, and perform gamut extension in $\Phi_{2}$, by adapting the method presented above in Section 3.1. Instead of having a single polarity of $\gamma$ for the whole image, we will consider different polarities for different pixels, with $\gamma>0$ for pixels with colors in $\Phi_{2}$ and $\gamma<0$ for pixels with colors in $\Phi_{1}$ and $\Psi$.

The steps of our method are:

1. Specify values for $\gamma$ for all $(R, G, B)$ points in $\Phi_{1}, \Phi_{2}$ and $\Psi$ : this can be expressed as a function or map $\Gamma: \mathbb{R}^{3} \longrightarrow \mathbb{R}$. 


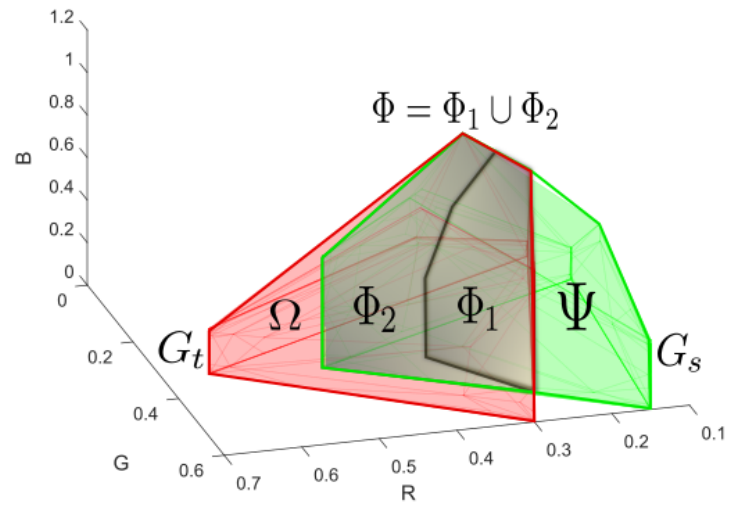

Figure 3: Source and target gamuts are partitioned into regions. In each of these regions the values for $\gamma$ are computed differently, and this determines the form of the gamut mapping process: whether it's going to be gamut reduction or gamut extension, and the amount of it.

2. Quantize these values into a reduced set $\Gamma_{q}=\left\{\gamma_{i}\right\}_{i=1, \ldots, N}$, so now the map becomes $\Gamma: \mathbb{R}^{3} \longrightarrow \Gamma_{q}$.

3. For each value $\gamma_{i}$ in $\Gamma_{q}$ run Eq. 2 on the three channels of $I_{s}$ until reaching steady state, obtaining a modified image $I_{s}^{i}$.

4. For each pixel $x$ such that the $\gamma$ value associated to color $I_{s}(x)$ is $\gamma_{i}$ (i.e. $\left.\Gamma\left(I_{s}(x)\right)=\gamma_{i}\right)$ then replace $I_{s}(x)$ with the color in position $x$ in the modified image $I_{s}^{i}(x)$.

A key part then is the first step, where values for $\gamma$ are assigned and the map $\Gamma$ is built. There is no unique solution for this, many choices are possible since in principle the only requirement is that $\gamma>0$ for pixels with colors in $\Phi_{2}$ and $\gamma<0$ for pixels with colors in $\Phi_{1}$ and $\Psi$. We have chosen to build $\Gamma$ in the following manner:

- If $z \in \Psi$, then:

$$
\Gamma(z)=\min \left(\gamma_{\min } \frac{d(z, \Phi)}{\max (d(\Psi, \Phi))}, \delta\right),
$$


$d(z, \Phi)=\min _{t \in \Phi} d(z, t)$, and $d(z, t)$ is the Euclidean distance between $z$ and $t$.

- If $z \in \Phi_{2}$, then:

$$
\Gamma(z)=\gamma_{\max } \frac{d_{\Omega, \Phi_{2}}(z)}{\max _{u \in \Phi_{2}} d_{\Omega, \Phi_{2}}(u)},
$$

where $\gamma_{\max }$ is a positive scalar, and $d_{\Omega, \Phi_{2}}(z)=\max _{t \in \Phi_{2}} d(t, \Omega)-d(z, \Omega)$.

\subsection{Implementation details}

For implementation purposes, we quantize each channel of the color space into 50 bins. Therefore, the total number of possible colors is $50^{3}=125000$. All 

the different bins. For the quantization of the $\Gamma$ map to $\Gamma_{q}$, we set the distance between two possible values in the quantized map to be 0.025 . This means that, for an image with $\gamma_{\min }=-1$ and $\gamma_{\max }=1$ the total number of different values in $\Gamma_{q}$ is 81 .

195 $\delta$, and we perform an analysis on their behavior in the results section. However, let us note here that in practice we mainly modify the parameters $\gamma_{\min }$ and $\gamma_{\max }$. Finally, regarding the parameters that our approach shares with the original model of Zamir et al. (the Gaussian kernel $w(x, y), \alpha, \beta, \Delta_{t}$ ) [10] we fix the recommended values originally set by the authors.

\section{Application to gamut mapping}

Let us start by showing the capabilities of our method in performing gamut mapping from an input gamut to a destination (target) gamut. To this end, in Figure 4 we show the effect of our method on two different images. From left to right we display the original image, our result, the result of only performing gamut reduction, and a plot showing the gamut of the original image (black), the gamut of our result (red), the input gamut (blue), and the destination gamut (green). In the top row, we show that our method performs as expected. The gamut in the region where the destination gamut is not present is reduced, while at the same time the gamut in the region where the original image does not cover much of the destination gamut is expanded. We want to highlight here that our method automatically learns if there is a need for gamut extension or gamut reduction. This feature presents a further advantage: In the case where no gamut reduction is needed, our method still performs a smooth gamut extension to improve the image. This can be seen in the bottom part of the figure. The major enhancement of the picture appears on the red of the hat.

We study the potential of our method for gamut mapping through the following experiment. We have considered 22 images from the Kodak database 
Table 1: Primaries used in the gamut mapping experiment.

\begin{tabular}{|c|c|c|c|c|c|c|}
\hline Gamut & \multicolumn{2}{|c|}{ R } & \multicolumn{2}{c|}{ G } & \multicolumn{2}{c|}{ B } \\
\hline & $\mathrm{x}$ & $\mathrm{y}$ & $\mathrm{x}$ & $\mathrm{y}$ & $\mathrm{x}$ & $\mathrm{y}$ \\
\hline sRGB & 0.64 & 0.33 & 0.30 & 0.60 & 0.15 & 0.06 \\
\hline Input gamut & 0.51 & 0.32 & 0.31 & 0.48 & 0.15 & 0.06 \\
\hline Destination gamut & 0.64 & 0.33 & 0.30 & 0.60 & 0.23 & 0.19 \\
\hline
\end{tabular}

[34] (they are shown in Figure 5). We have reduced them to a smaller gamut,

that we call the input gamut, via 10. These reduced images are the input images for the experiment. Then, we have defined a second gamut, that we call the destination gamut, not fully encompassed in the input gamut. The goal of the experiment is to perform gamut mapping from the input images to the destination gamut. The primaries of the gamuts are shown in Table 1, and a plot showing the gamuts is presented in Figure 6 .

Hereinafter, all the results we will present in this section have been obtained with a fixed set of parameters, in particular $\gamma_{\max }=0.4, \gamma_{\min }=-1, \delta=\frac{\gamma_{\min }}{4}$, and $\tau=0.5$. The map $\Gamma$ has been obtained in the $x$ and $y$ components of the $x y Y$ color space. Eq. 2 is applied to the $a$ and $b$ channels of the Lab color space for extension, to RGB for reduction and using the parameters mentioned in [35] and [10] respectively. The $Y$ component of the $x y Y$ color space of the final image has been replaced by the $Y$ component of the original image (this change does not affect the xy chromaticity diagram).

We compare our method to two different approaches: First, we just apply the gamut reduction method of Zamir et al. [10] between the input gamut and the destination gamut and second, we concatenate a well-known gamut extension method (SDS [6]) to go back to the full sRGB gamut followed by a well-known gamut reduction method (Alsam and Farup [36]) to go from sRGB to the destination gamut. In Figure 7 we show the result of concatenating SDS and Alsam and Farup methods on the left, the result of Zamir et al. reduction in 

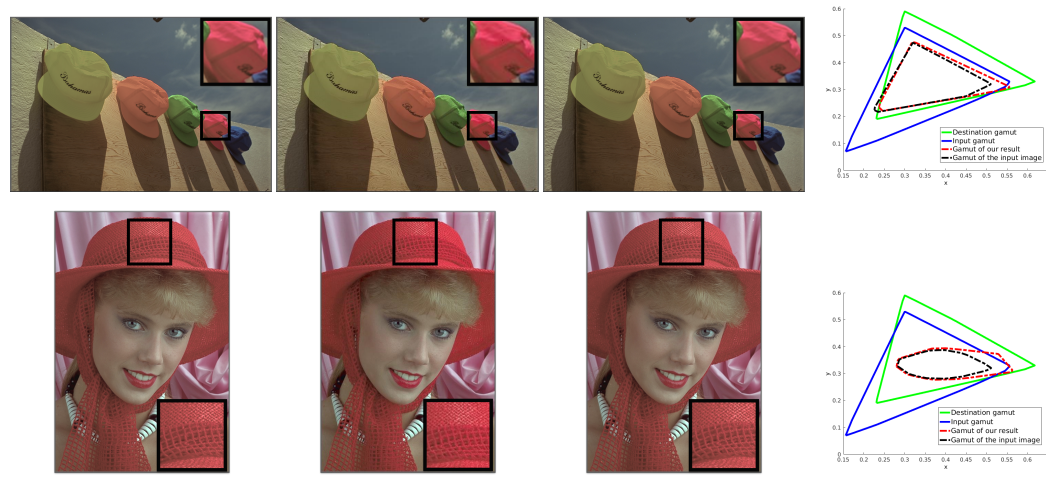

Figure 4: An example of our method for gamut mapping. From left ro right: Original image, our result, result of only performing gamut reduction. Plots in the right column show the gamut of the original image (black), the gamut of our result (red), the input gamut (blue), and the destination gamut (green). In this figure we have used the following parameters. Top row: $\gamma_{\max }=0.45, \gamma_{\min }=-0.4, \delta=\frac{\gamma_{\min }}{4}$, and $\tau=0.5$. Bottom row: $\gamma_{\max }=0.45, \gamma_{\min }=-1$, $\delta=\frac{\gamma_{\min }}{4}$, and $\tau=0.5$.

the center, and our result on the right. Our result looks more natural, especially when focusing the attention on the blue of the sky, the green of the grass, or the red of the house. In these cases the reduction approach provides results that lack saturation, while the concatenation method produces over-saturated colors that are not realistic.

We have run a psychophysical evaluation to compare the three aforementioned methods for the 22 images of Figure 5 The experiment was performed as a pair comparison test where 7 observers (all the observers were color image processing experts, but none of them are the authors of this paper) were asked for their preferred image. Users were presented the results of two different methods side by side and they were forced to select one of the results (i.e. it was a forced-choice experiment, ties between the methods were not allowed). The experiment was performed in a dark room and the background of the screen was set to a mid-gray. We have extracted the results of the experiment using Case 5 of the Thurstone 255 Law of Comparative Judgement [37.

Results are presented in Table 2 and show that our method outperforms the 


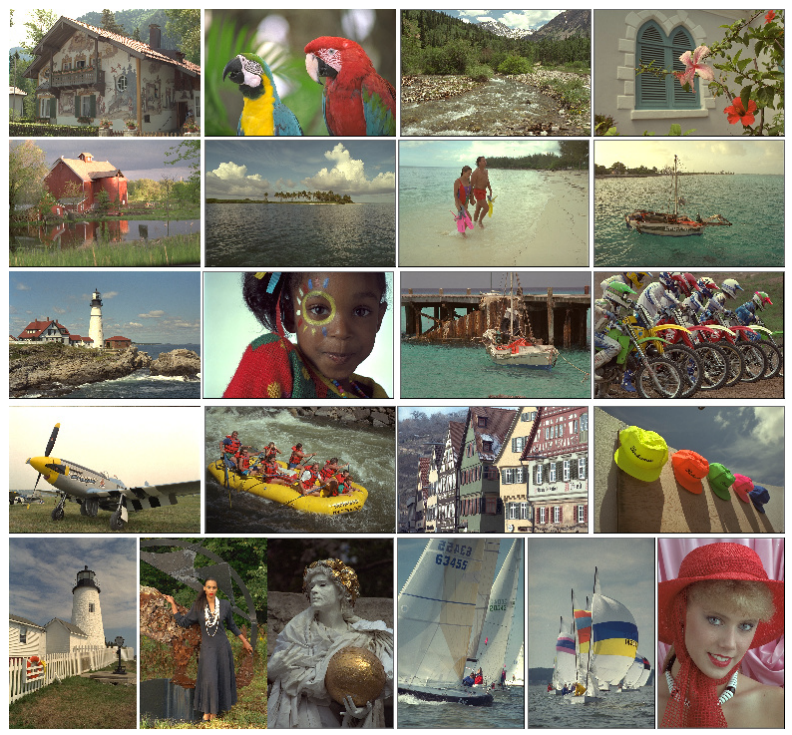

Figure 5: Images used in the gamut mapping experiment.

other two, where the reduction method of Zamir et al. outperforms the hybrid approach. Note that the Zamir et al. method adapts to the image content, while we are fixing the values of $\gamma_{\max }, \gamma_{\min }, \tau$, and $\delta$ for all the images. Hence, even an image-based manner.

We have also computed a blind image quality metric based on naturalness constraints, the "Naturalness image quality evaluation" (NIQE) 38] on the same set of images. Results in terms of the average NIQE value for the 22 images of

Our method outperforms the other two contending approaches. Furthermore, the ranking of the algorithms is the same as in the psychophysical experiment.

\section{Application to image processing: Color coherence}

In this section we apply our method to the problem of color coherence. Color 270 coherence aims at modifying a source image in order to make its colors consistent with the colors of an unrelated second image but without modifying the general 


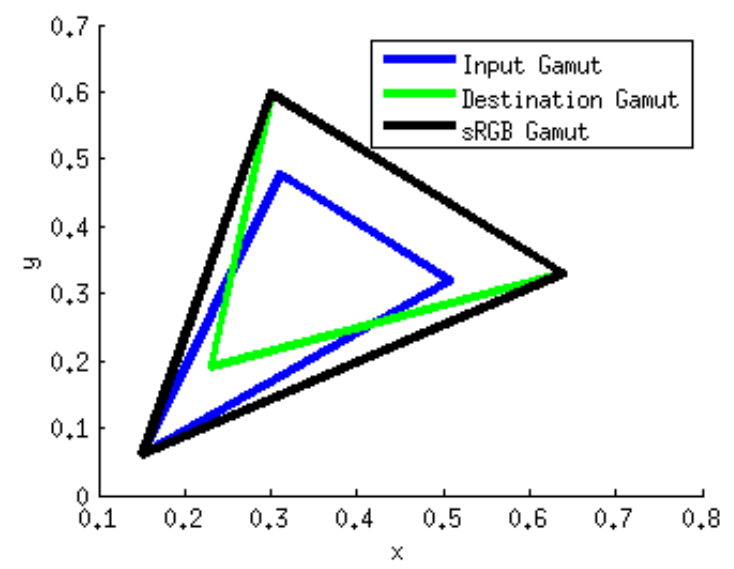

Figure 6: Gamuts used for the gamut mapping experiment.

Table 2: Thurstone Law Case 5 results for the gamut mapping experiment. Average for the 22 images of Figure 5

\begin{tabular}{|c|c|c|}
\hline Zamir et al. & Std. GE + Std. GR & Ours \\
\hline 0.1024 & -0.5003 & $\mathbf{0 . 3 9 8 0}$ \\
\hline
\end{tabular}

look of the first image. This is different to general color transfer where the goal is to have the same color statistics in the source and the target images. In this section we have used image-based parameters for $\gamma_{\min }$ and $\gamma_{\max }$ (see Appendix), and we have set $\delta=\frac{\gamma_{\min }^{2}}{2}$ and $\tau=0.35$ for all the images. The map $\Gamma$ has been obtained in RGB color space and Eq. 2 is applied to the $a$ and $b$ channels of the Lab color space for extension, to RGB for reduction and using the parameters mentioned in [10, 35].

In Figure 8 we present a detailed result for our approach. The first column shows the source (top) and the reference (bottom) images, and the second column presents our result with the map of the gamma values used for the image.

Four different examples of our results can be seen in Figure 9 In this figure, the first column shows the source image, the second column shows the target image, and the third column shows our results. We want the reader to focus on 

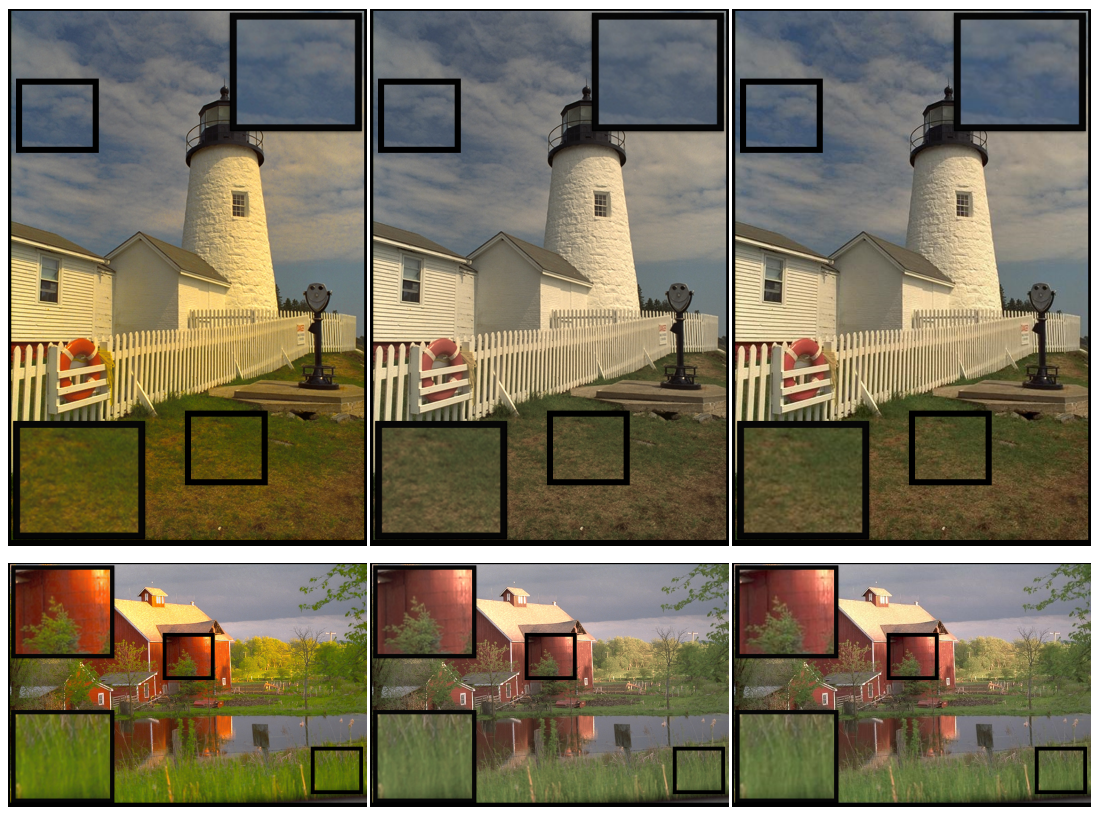

Figure 7: Comparison between: Left, a concatenation of a standard gamut extension method (SDS [6]) and a standard gamut reduction method (Alsam and Farup [8]). Center, the reduction of Zamir et al. [10. Right, our result.

the green of the leaves, the orange of the trees and the blue of the sky in the first row, the blue of the sky and the red of the flowers in the second row, the green of the map in the third row, and finally, the fogging effect in the fourth row.

In Figure 10 we see how our method compares to four of the most well-known color transfer methods. From left to right we show: source image, target image, Kotera [26, Pitié et al. [25, Reinhard et al. 24], Xiao et al. [39], and our results. We can see that our method is able to move towards the color gamut of the target image whilst retaining the feel of the original image. On the contrary, pure color transfer methods generate images that are in general not pleasant in appearance. To quantitatively examine these results we have computed the NIQE quality metric [38] for the images in this Figure. The results of this metric are presented in Table 4 , where smaller values represent higher accuracy. Our method outperforms the other approaches. 
Table 3: Quantitative results for the images used in the psychophysical experiment. Average for the 22 images of Figure 5

\begin{tabular}{|c|c|c|c|}
\hline Metric & Zamir et al. & Std. GE + Std. GR & Ours \\
\hline NIQE [38] & 2.97 & 3.11 & $\mathbf{2 . 7 2}$ \\
\hline
\end{tabular}
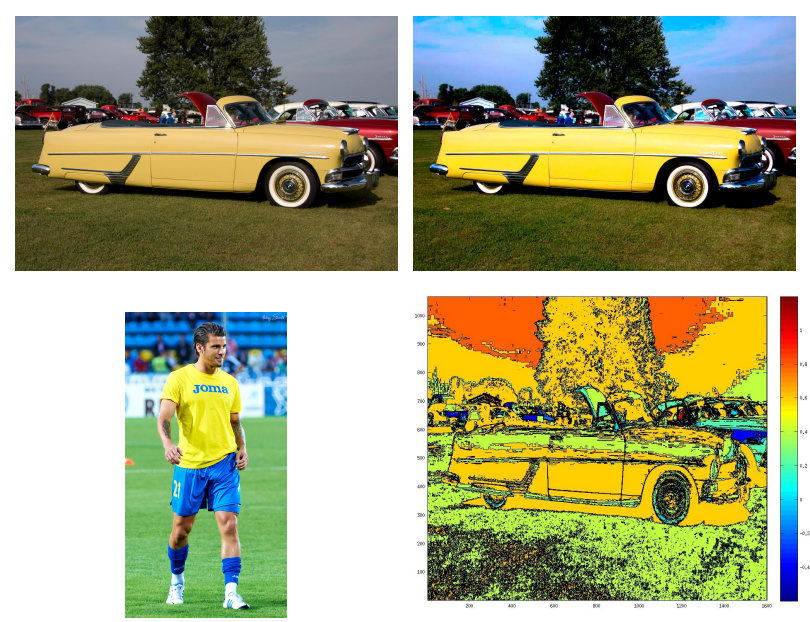

Figure 8: An example of our color coherence approach. Two left images: Source (top) and target (bottom) images. Two right images: Our result (top) and the corresponding gamma values (bottom).

Finally, let us note that our color coherence approach may also be applied for other problems, such as semantic transfer [40] or any other one-to-many color transfer. In this case, the idea is to construct the $\Gamma$ map based on either the color histogram or the color gamut of the images we want our image to look like. A first example of this application is shown in Figure 11 where we can see the original image (left) and the result of our method when considering a target gamut composed by the first 50 images that appear in a search engine for the concept 'strawberry' in the top image, the concept 'banana' in the center image, and the concept 'warm' in the bottom image (right). 

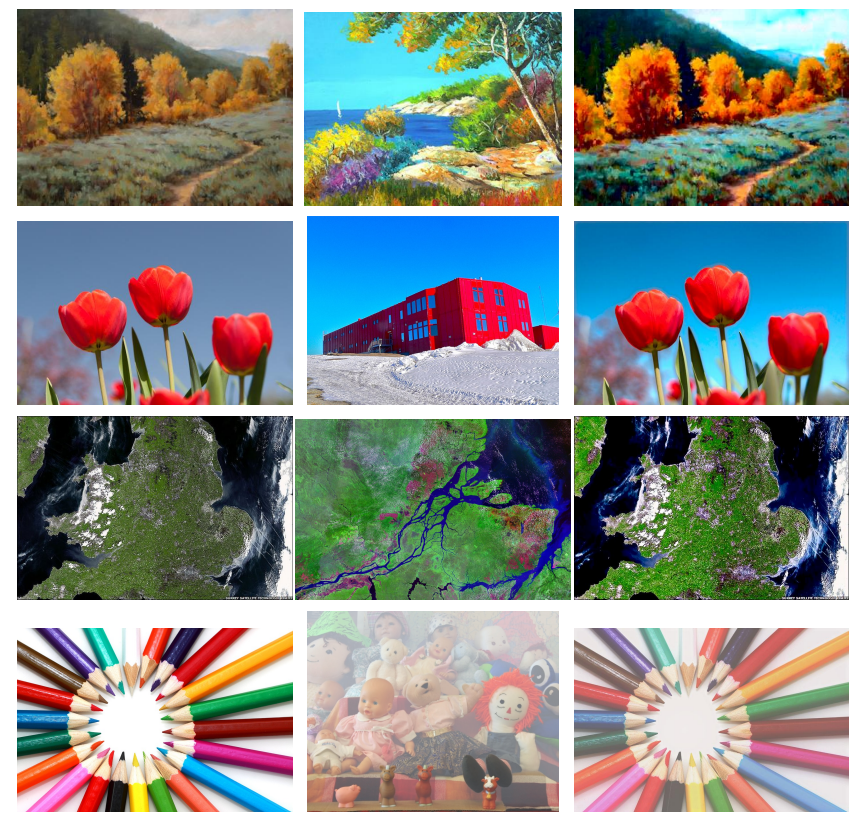

Figure 9: An example of our color coherence approach. From left to right: Source image, target image, our results.

Table 4: Quantitative results computed as the average for all the images of Figure 8.

\begin{tabular}{|c|c|c|c|c|c|}
\hline Metric & {$[25]$} & {$[26]$} & {$[24$} & {$[\underline{39}$} & Ours \\
\hline NIQE [38] & 3.34 & 3.53 & 3.91 & 3.56 & $\mathbf{3 . 0 5}$ \\
\hline
\end{tabular}

\subsection{On the analysis of the parameters}

Let us now analyze the importance of the different parameters. It is not straightforward to automatically select these parameters, since they strongly depend not only on the source and target images, but also on the aesthetic preference of the user. Let us start by analyzing the importance of $\gamma_{\max }$ and $\gamma_{\min }$. In Figure 12 we show in the first row a source and a target image. Then, in the other four rows, we show different results of the method by modifying both $\gamma_{\max }$ and $\gamma_{\min }$. In particular, the value of $\gamma_{\min }$ changes over the different rows in a decreasing manner (from -0.25 in the upper row to $-0.50,-0.75$ and -1 in the 
other rows respectively). Conversely, the value of $\gamma_{\max }$ is increased column-wise, (from 0.25 for the first column, to $0.5,0.75$ and 1 for the others). The rest of parameters are equal to those described for Figure 10. We can observe that as the parameter $\gamma_{\min }$ gets reduced, the blue color of the sky loses its prominence, as it is a color not appearing in the target image. Conversely, as the value of $\gamma_{\max }$ increases, the yellow color gets extended towards the yellow of the bananas.

The role of the parameter $\tau$ is studied in the top row of Figure 13 . In this row, we can see from left to right, the source image, the target image, and four different results for our method. The value of $\tau$ moves between 0.75 (in the leftmost result) and 0.15 (in the rightmost result). The rest of parameters are equal to those described for Figure 10. We can clearly observe that, as the value of $\tau$ increases the image is less extended,-as expected (see the explanation of Equation 9).

Finally, the role of the parameter $\delta$ is analyzed in the bottom row of Figure 33013 In this row, from left to right, we have the source image, the target image, and four results of our method generated by varying $\delta$ between 0 and -1 in equal decrements. The rest of parameters are equal to those used in Figure 10. As expected, when we decrease the value of $\delta$ most of the colors become closer to the achromatic axis. The reason for this behavior is that $\delta$ is the parameter that controls the amount of reduction given to those colors of the source image that are already in the target gamut but, at the same time, are the closest to the source colors that lie outside the target gamut (i.e. those colors at the intersection of $\Psi$ and $\Phi$ ). Therefore, $\delta$ can be used to modulate the amount of gamut reduction once $\gamma_{\min }$ has already been fixed.

\section{Conclusions}

In this work we introduced a method to simultaneously perform gamut reduction and extension. To the best of our knowledge, this is the first time that a method of this kind has been proposed. We showed that our approach is useful for the problems of gamut mapping and color coherence. Further work 

and with the semantic transfer problem [40] (outlined in Figure 11).

\section{Acknowledgements}

This work is supported by the European Research Council, Starting Grant ref. 306337, by the Spanish government FEDER Fund, grant ref. TIN201571537-P(MINECO/FEDER,UE), and by the Icrea Academia Award. The work of J. Vazquez-Corral was supported by the Spanish government under Grant IJCI-2014-19516

[1] J. Morovič, Color gamut mapping, Vol. 10, Wiley, 2008.

[2] T. AA, http://www.androidauthority.com/best-of-android-2015-display660168/ (December 2015, accessed 1st August 2016).

[3] Brelco, http://www.brelco.it/prodotti/oled-en.htm (accessed 1st August 2016).

[ [4] S. J. Dain, B. Kwan, L. Wong, Consistency of color representation in smart

n phones, J. Opt. Soc. Am. A 33 (3) (2016) A300-A305. doi:10.1364/JOSAA. 33.00A300.

URL http://josaa.osa.org/abstract.cfm?URI=josaa-33-3-A300

[5] G. M. Murch, J. M. Taylor, Color in computer graphics: Manipulating and matching color, Eurographics Seminar: Advances in Computer Graphics V (1989) 41-47.

365 [6] J. Laird, R. Muijs, J. Kuang, Development and evaluation of gamut extension

口 algorithms, Color Research \& Application 34 (6) (2009) 443-451. doi: $10.1002 / \mathrm{col} .20537$

URL http://dx.doi.org/10.1002/col.20537

[7] K. Jang, H. Cho, H. Kang, B. Kang, A color gamut mapping system using 
device, in: Proc. of International Conference on Future Information \& Communication Engineering, 2013, pp. 559-568.

[8] A. Alsam, I. Farup, Spatial colour gamut mapping by orthogonal projection of gradients onto constant hue lines, in: Proc. of 8th International Symposium on Visual Computing, 2012, pp. 556-565.

[9] I. Farup, C. Gatta, A. Rizzi, A multiscale framework for spatial gamut mapping, IEEE Transactions on Image Processing 16 (10) (2007) 2423-2435.

[10] S. Zamir, J. Vazquez-Corral, M. Bertalmio, Gamut mapping in cinematography through perceptually-based contrast modification, IEEE Journal of Selected Topics in Signal Processing 8 (3) (2014) 490-503.

[11] R. S. Gentile, E. Walowitt, J. P. Allebach, A comparison of techniques for color gamut mismatch compensation, Journal of Imaging Technology 16 (1990) 176-181.

[12] J. J. McCann, A spatial colour gamut calculation to optimize colour appearance, in: Colour Image Science: Exploiting Digital Media, 2002, pp. 213-233.

[13] F. Schweiger, T. Borer, M. Pindoria, Luminance-preserving colour conversion, in: SMPTE Annual Technical Conference and Exhibition, 2016, pp. $1-9$.

[14] J. Pytlarz, K. Thurston, D. Brooks, P. Boon, R. Atkins, Real time crossmapping of high dynamic range images, IET Conference Proceedings.

[15] Y. Li, G. Song, H. Li, A multilevel gamut extension method for wide gamut displays, in: Proc. of International Conference on Electric Information and Control Engineering (ICEICE),, 2011, pp. 1035-1038.

[16] X. Chen, Investigation of gamut extension algorithms, Master's thesis, University of Derby, UK (2002). 
[17] H. Anderson, E. Garcia, M. Gupta, Gamut expansion for video and image sets, in: Proc. of the 14th International Conference of Image Analysis and Processing - Workshops, 2007, pp. 188-191.

[18] B. H. Kang, J. Morovič, M. R. Luo, M. S. Cho, Gamut compression and extension algorithms based on observer experimental data, ETRI journal 25 (3) (2003) 156-170.

[19] H. Pan, S. Daly, A gamut-mapping algorithm with separate skin and nonskin color preference controls for wide-color-gamut TV, SID Symposium Digest of Technical Papers 39 (1) (2008) 1363-1366.

[20] X. Meng, G. Song, H. Li, A human skin-color-preserving extension algorithm for wide gamut displays, in: Proc. of International Conference on Information Technology and Software Engineering, Lecture Notes in Electrical Engineering, 2013, pp. 705-713.

[21] S. E. Casella, R. L. Heckaman, M. D. Fairchild, Mapping standard image content to wide-gamut displays, in: Color and Imaging Conference, 2008, pp. 106-111.

[22] R. L. Heckaman, J. Sullivan, Rendering digital cinema and broadcast TV content to wide gamut display media, SID Symposium Digest of Technical Papers 42 (1) (2011) 225-228. doi:10.1889/1.3621279. URL http://dx.doi.org/10.1889/1.3621279

[23] S. W. Zamir, J. Vazquez-Corral, M. Bertalmío, Gamut extension for cinema, IEEE Transactions on Image Processing 26 (4) (2017) 1595-1606.

[24] E. Reinhard, M. Ashikhmin, B. Gooch, P. Shirley, Color transfer between images, IEEE Comput. Graph. Appl. 21 (5) (2001) 34-41.

[25] F. Pitié, A. Kokaram, R. Dahyot, Automated colour grading using colour distribution transfer, Computer Vision and Image Understanding 107 (1) (2007) 123-137. 
[26] H. Kotera, A scene-referred color transfer for pleasant imaging on display, in: Proceedings of IEEE ICIP 2005, 2005, pp. 5-8.

[27] Y. HaCohen, E. Shechtman, D. Goldman, D. Lischinski, Non-rigid dense correspondence with applications for image enhancement, ACM Transactions on Graphics (TOG) 30 (4) (2011) 70.

[28] O. Frigo, N. Sabater, J. Delon, P. Hellier, Motion driven tonal stabilization, IEEE Transactions on Image Processing 25 (11) (2016) 5455-5468. doi: 10.1109/TIP.2016.2601267.

[29] J. Vazquez-Corral, M. Bertalmio, Color stabilization along time and across shots of the same scene, for one or several cameras of unknown specifications, Image Processing, IEEE Transactions on 23 (10) (2014) 4564-4575. doi: 10.1109/TIP.2014.2344312

[30] J. Vazquez-Corral, M. Bertalmío, Log-encoding estimation for color stabilization of cinematic footage, in: 2016 IEEE International Conference on Image Processing, ICIP 2016, Phoenix, AZ, USA, September 25-28, 2016, 2016, pp. 3349-3353. doi:10.1109/ICIP.2016.7532980. URL https://doi .org/10.1109/ICIP.2016.7532980

[31] Y. HaCohen, E. Shechtman, D. B. Goldman, D. Lischinski, Optimizing color consistency in photo collections, ACM Transactions on Graphics (TOG) 32 (4) (2013) 38.

[32] M. Bertalmío, V. Caselles, E. Provenzi, A. Rizzi, Perceptual color correction through variational techniques, IEEE transactions on image processing: a publication of the IEEE Signal Processing Society 16 (4) (2007) 1058-1072.

[33] M. Bertalmio, V. Caselles, E. Provenzi, Issues about retinex theory and contrast enhancement, International Journal of Computer Vision 83 (1) (2009) 101-119.

${ }_{450}$ [34] http://r0k.us/graphics/kodak/ (accessed 6th July 2017). 
[35] S. W. Zamir, J. Vazquez-Corral, M. Bertalmío, Gamut extension for cinema: Psychophysical evaluation of the state of the art, and a new algorithm, in: Proc. of IS\&T/SPIE Human Vision and Electronic Imaging XX, 2015.

[36] A. Alsam, I. Farup, Colour gamut mapping as a constrained variational problem, in: Proc. of 16th Scandinavian Conference on Image Analysis, 2009, pp. 109-118.

[37] L. L. Thurstone, A law of comparative judgment, Psychological Review 34 (4) (1927) 273-286.

[38] A. Mittal, R. Soundararajan, A. C. Bovik, Making a "completely blind" image quality analyzer., IEEE Signal Process. Lett. 20 (3) (2013) 209-212.

[39] X. Xiao, L. Ma, Color transfer in correlated color space, in: Proceedings of the 2006 ACM International Conference on Virtual Reality Continuum and Its Applications, VRCIA '06, ACM, New York, NY, USA, 2006, pp. 305-309. doi:10.1145/1128923.1128974.

${ }_{465}$ [40] A. Lindner, S. Süsstrunk, Semantic-Improved Color Imaging Applications: It Is All About Context, IEEE TMM, Transactions on Multimedia PP (99).

\section{Appendix: Parameters used in the experiments of section $\mathrm{V}$}

The parameters $\gamma_{\min }$ and $\gamma_{\max }$ used in the different examples of section $\mathrm{V}$ are shown in Table 5 . 
Table 5: Parameters $\gamma_{\min }$ and $\gamma_{\max }$ used in our experiments for color coherence.

\begin{tabular}{|c|c|c|}
\hline Image & $\gamma_{\max }$ & $\gamma_{\min }$ \\
\hline Figure 8 & 1.2 & -0.6 \\
Figure 9, row 1 & 0.8 & -0.8 \\
Figure 9, row 2 & 1 & -0.5 \\
Figure 9, row 3 & 0.75 & -0.75 \\
Figure 9, row 4 & 1 & -1 \\
Figure 10, row 1 & 1.25 & -1 \\
Figure 10, row 2 & 1 & -1 \\
Figure 10, row 3 & 1 & -0.5 \\
Figure 10, row 4 & 1 & -1 \\
Figure 10, row 5 & 1.75 & -1.75 \\
Figure 10, row 6 & 0.5 & -0.5 \\
Figure 10, row 7 & 1.3 & -0.5 \\
Figure 10, row 8 & 1 & -0.75 \\
Figure 10, row 9 & 0.9 & -0.75 \\
Figure 10, row 10 & 0.9 & -0.9 \\
\hline
\end{tabular}




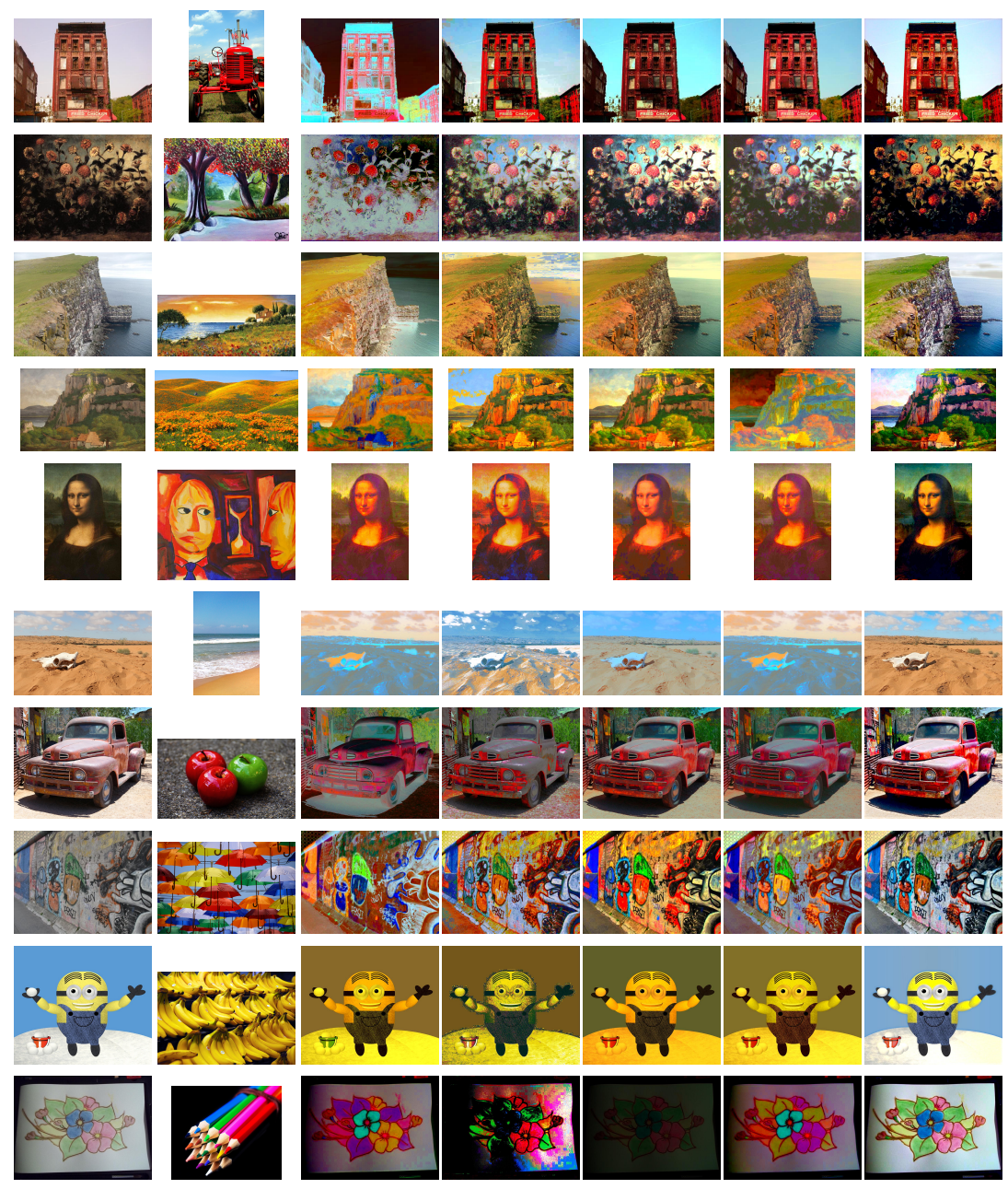

Figure 10: A comparison of our method versus color transfer methods. From left to right column: Source image, target image, Kotera [26], Pitie et al. [25], Reinhard et al. 24], Xiao et al. 39, our method. 

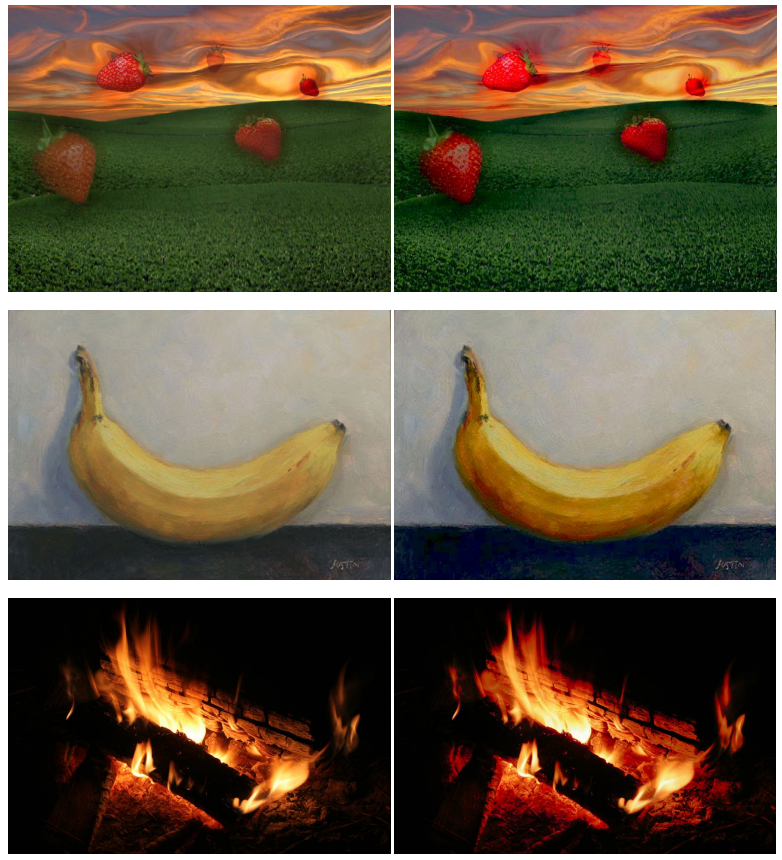

Figure 11: Example of our method when applied to semantic transfer. Left: original image, right: our result for the terms 'strawberry' (top), 'banana' (middle), and 'warm' (bottom). 

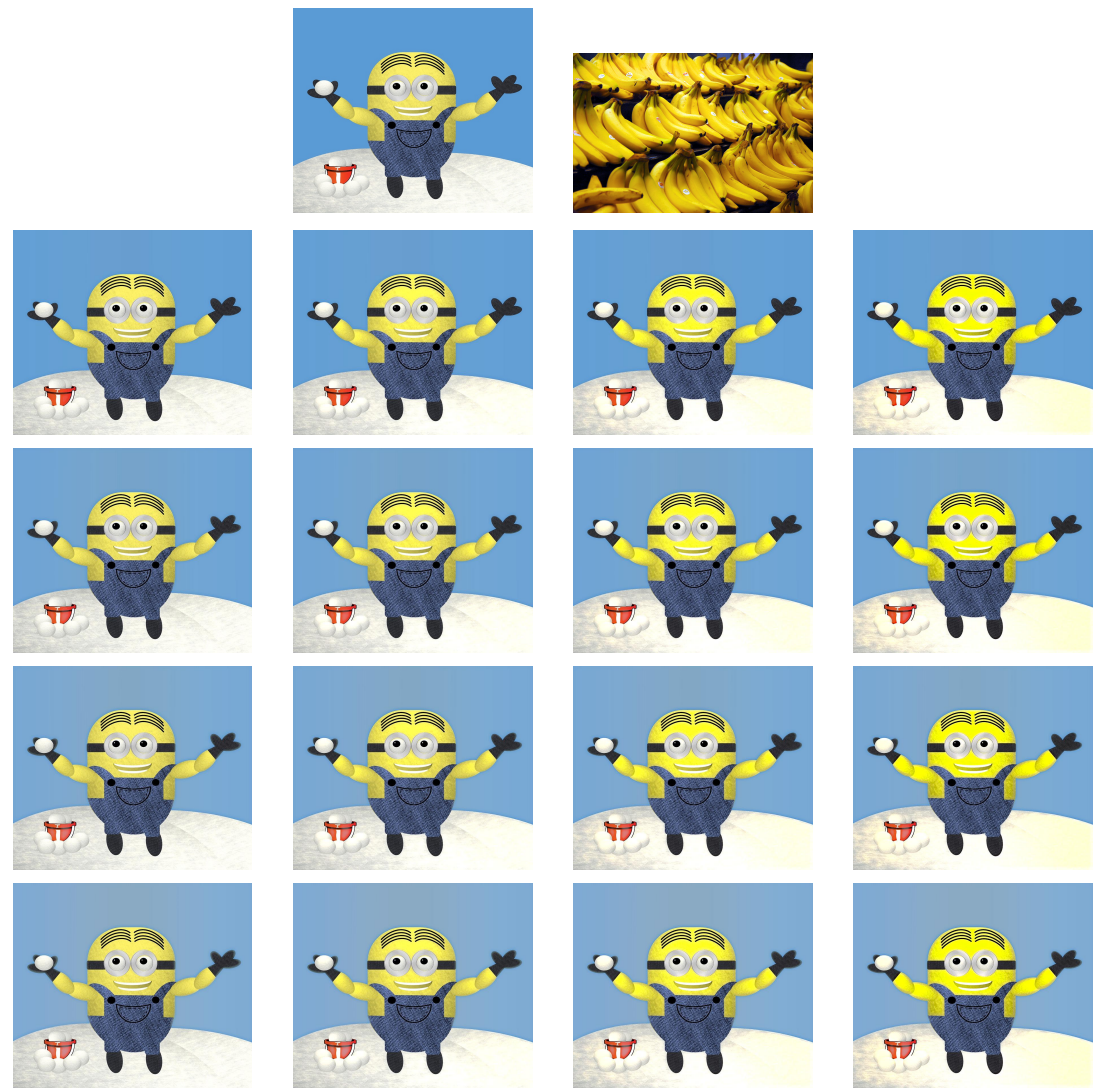

Figure 12: A visual example of the behaviour of the parameters $\gamma_{\max }$ and $\gamma_{\min }$. Top row: left is the source image and right is the target image. Second to fifth rows represent a modification in the $\gamma_{\text {min }}$ parameter, being the values $-0.25,-0.5,-0.75,-1$ from top to bottom. For these four rows, each column represents a modification in the $\gamma_{\max }$ parameter, being the values 0.25 , $0.5,0.75,1$ from left to right. The other parameters are the same as in Figure 10 


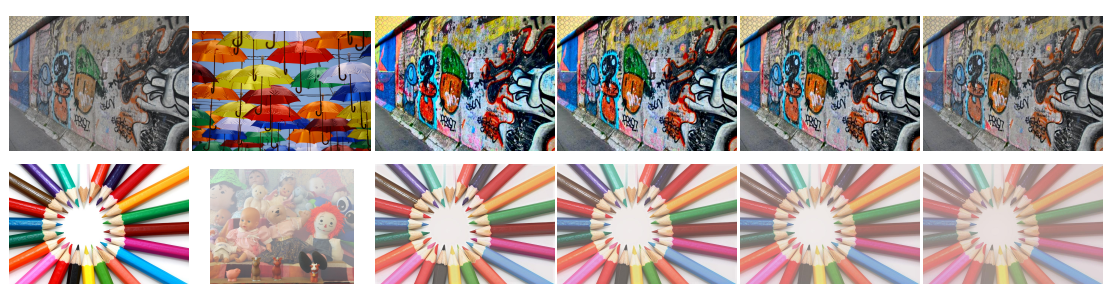

Figure 13: Top row: A visual example of the behaviour of the parameter $\tau$. From left to right: source image, target image, $\tau=0.15, \tau=0.35, \tau=0.55$, and $\tau=0.75$. In this example, the other parameters are the same as in Figure 10

Bottom row: A visual example of the behaviour of the parameter $\delta$. From left to right: source image, target images, $\delta=0, \delta=\frac{\gamma_{\min }}{3}, \delta=\frac{2 \gamma_{\min }}{3}$, and $\delta=\gamma_{\min }$. In this example, $\gamma_{\min }$ and the rest of parameters are the same as in Figure 10 